\title{
A layered history
}

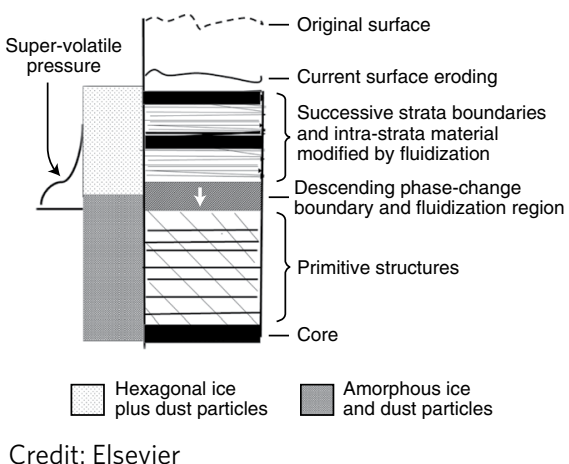

Observations from the high-resolution camera on board Rosetta have shown that the two lobes of comet 67P/ChuryumovGerasimenko's nucleus exhibit independent systems of global-scale stratification, indicating that the two parts of $67 \mathrm{P}$ merged after the stratification mechanism set in. Determining the physics behind the strata is thus key to understanding the history of $67 \mathrm{P}$ and possibly of all the Jupiter-family comets (JFCs). Michael Belton and colleagues put together images of $67 \mathrm{P}$ - particularly its Hathor cliff region - and 9P/Tempel-1 with Tancredi and colleagues' thermophysical model (Astron. Astrophys. 286, 659-682; 1994) to reconstruct a coherent scenario (pictured schematically).

The layers appear only at a late stage of a comet's history, during its Centaur phase an active asteroid orbiting in the outer Solar
System. When the Centaur is close enough to the Sun (at heliocentric distance $\sim 8$ $\mathrm{au}$ ), it becomes warm enough to onset the crystallization of its amorphous ice, starting from the surface and propagating inwards. The crystallization proceeds in two repeated steps: a several-years-long quiescent mode generating the strata, followed by a quick active burst lasting a few weeks, which creates the intra-strata material. Radial outgassing of volatiles, particularly $\mathrm{CO}$, controls the crystallization front propagation and gives the stratification its ordered appearance. The whole process could have crystallized the whole comet in a few millennia and is potentially present in all JFCs.

Interestingly, while the authors suggest a primeval bilobate shape of $67 \mathrm{P}$, which broke up at the beginning of crystallization and merged again only after the two parts completed their independent stratification, this scenario is also compatible with an independent formation and evolution of the lobes followed by a late collision, as suggested by Schwartz et al. (Nat. Astron. 2, 379-382; 2018).

This Research Highlight is a tribute to the outstanding career and contribution to planetary science of Mike Belton (1934-2018).

Luca Maltagliati

Published online: 27 June 2018

https://doi.org/10.1038/s41550-018-0528-7 